\title{
Effects of antenatal lipopolysaccharide and postnatal hyperoxia on airway reactivity and remodeling in a neonatal mouse model
}

\author{
Arij Faksh', Rodney D. Britt Jr², Elizabeth R. Vogel ${ }^{2}$, Ine Kuipers², Michael A. Thompson², Gary C. Sieck ${ }^{3}$, Christina M. Pabelick ${ }^{2,3}$, \\ Richard J. Martin ${ }^{4}$ and Y. S. Prakash ${ }^{2,3}$
}

BACKGROUND: Antenatal inflammation and preterm birth are associated with the development of airway diseases such as wheezing and asthma. Utilizing a newborn mouse model, we assessed the effects of maternal inflammation and postnatal hyperoxia on the neonatal airway.

METHODS: Pregnant C57/Bl6 dams were injected with lipopolysaccharide (LPS) or saline on embryonic day 16. Offspring were placed in room air or hyperoxia $\left(50 \% \mathrm{O}_{2}\right)$ for $7 \mathrm{~d}$ and then returned to normoxia. Airway mechanics, histology, and laser capture micro-dissection (LCM) were performed.

RESULTS: At postnatal day 21, maternal LPS- and 50\% $\mathrm{O}_{2}-$ exposed pups exhibited increased resistance and decreased compliance compared to $21 \% \mathrm{O}_{2}$ pups; however their effects were not synergistic. LPS and hyperoxia each increased the thickness of airway smooth muscle (ASM), but not the airway epithelial layer. Structural changes were largely limited to the conducting airways. Upregulation of inflammatory markers in the lung was observed at birth. LCM revealed increased collagen-3, transforming growth factor $\beta$, and connective tissue growth factor expression with LPS and hyperoxia within the ASM layer.

CONCLUSION: These novel studies provide functional, structural, and molecular evidence that antenatal inflammation is detrimental to the developing airway. Exposure to moderate hyperoxia does not exacerbate LPS effects on the airway.

$P^{\prime}$ reterm birth, defined as delivery prior to 37 weeks gestational age, remains a significant public health concern worldwide, affecting up to $18 \%$ of all pregnancies $(1,2)$. Preterm infants are at high risk of impaired lung development and function (3). Maternal complications during pregnancy due to infection and subsequent inflammation are a common cause of preterm birth. Maternal infection and/or inflammation can arise from both intrauterine sources such as chorioamnionitis, caused by bacterial or viral infection within the fetal membrane or extrauterine sources such as systemic maternal inflammation from pneumonia, pyelonephritis, asymptomatic bacteriuria, or appendicitis $(1,2,4,5)$. Bacterial or viral infection of the amniotic fluid, fetal membranes, placenta, or uterus can occur (5). Interestingly, even a transient chorio-decidual infection induces cytokine production in the amniotic fluid, which can lead to a fetal inflammatory response without overt infection of amniotic fluid or preterm labor (6).

Inflammation during the perinatal period instigates preterm birth, as previously demonstrated in human and animal investigations $(7,8)$. Given their immature lungs, preterm neonates commonly require supplemental oxygen and ventilator support in the neonatal intensive care environment. Such necessary interventions impair lung growth substantially, contributing to airway disease (9). While clinical practice has evolved toward limiting the extent of hyperoxia, and the use of noninvasive ventilator support, recent studies demonstrate that infants receiving even moderate oxygen remain at risk of conducting/bronchial airway disease (including asthma), which differs from the alveolar simplification pathology of bronchopulmonary dysplasia (10). However, the mechanisms by which exposure to antenatal inflammation and subsequent hyperoxia exposure during the neonatal period influence postnatal bronchial airway structure and function have not been firmly established (4).

Clinical studies demonstrate increased risk of childhood asthma in babies born to mothers with respiratory tract infections, febrile infectious diseases, urinary tract infections, or vaginitis in pregnancy, suggesting that maternal inflammation can contribute to development of wheezing and asthma in preterm infants $(11,12)$. Hyperoxia also represents a major contributing factor to lung disease, as supported by animal studies showing increased inflammation, reduced alveolar development, and altered bronchial airway structure and function (13).

Lipopolysaccharide (LPS), a potent proinflammatory stimulus, has been used in numerous animal models of perinatal inflammation $(14,15)$. Prior studies have utilized intra-amniotic 
LPS administration to model chorioamnionitis, and showed resultant neonatal inflammation of the lung, as well as other organ systems, resulting in neonatal lung injury, remodeling, and pulmonary hypertension (15-18). However, the effects of LPS on bronchial airways are less understood, but are key to knowing how inflammation contributes to wheezing. A major feature of airway remodeling is enhanced extracellular matrix (ECM) (19) produced by airway smooth muscle (ASM) and fibroblasts (20). In addition to regulating airway tone and contractility, ASM also releases and responds to cytokines and growth factors. From the remodeling perspective, antenatal inflammation promotes transforming growth factor (TGF $\beta$ ) expression, and alters expression of other mediators such as connective tissue growth factor (CTGF) $(17,21)$. Additionally, TGF $\beta$ and factors such as CTGF enhance expression of proteins involved in remodeling such as collagen-1 and collagen-3 $(22,23)$. Similar pathways are also implicated in the inflammation and remodeling resulting from postnatal hyperoxia exposure $(15,16)$. Thus, understanding the effects of perinatal inflammation and/or hyperoxia on the developing bronchial airway, particularly ASM, becomes important.

The goal of the current study was to explore the effects of systemic maternal inflammation and postnatal hyperoxia on developing bronchial airway function and structure. A newborn mouse model was used to model acute systemic maternal inflammation that is commonly confronted by premature neonates that go on to receive oxygen supplementation postnatally. We hypothesized that compared to saline/room air $(21 \%$ $\mathrm{O}_{2}$ ) exposed pups, LPS/hyperoxia $\left(50 \% \mathrm{O}_{2}\right)$-exposed mouse pups will demonstrate the greatest increase in airway reactivity and remodeling.

\section{RESULTS}

\section{Body Weights}

Maternal body weight was assessed at the time of the IP injection on E16, and then daily until spontaneous delivery. LPS administration did not adversely affect maternal weight gain suggesting that maternal failure to thrive was not an issue (data not shown). Neonatal weights were assessed at birth, day of life (DOL) 5, 14, and 21. At birth (DOL 1), there were no significant differences between the weights of pups from LPS vs. saline-exposed mothers (Table 1). At DOL 5, the hyperoxiaexposed treatment groups showed a significantly lower weight as compared to Saline/RA exposed pups (Table 1). At DOL 14, there were no differences between any of the treatment groups with respect to body weight (Table 1). Interestingly, at DOL 21, body weight was significantly lower in pups exposed to LPS/ RA and LPS $/ \mathrm{O}_{2}$ compared to Saline/RA control, but no significant differences in body weight was observed between control pups and the Saline $/ \mathrm{O}_{2}$ treatment group (Table 1).

\section{Respiratory Mechanics}

Compared to Saline/RA mice, LPS/RA and $\mathrm{LPS} / \mathrm{O}_{2}$ mice demonstrated increased total airway resistance $\left(\mathrm{R}_{\mathrm{L}} ; \mathrm{cmH}_{2} \mathrm{O} . \mathrm{s} / \mathrm{ml}\right.$; entire respiratory system including airway, lungs, and chest wall) and reduced dynamic compliance at baseline (Figure 1;
Table 1. Neonatal body weights grams at DOL 1 (birth), DOL 5, 14, and 21

\begin{tabular}{lcccc}
\hline $\begin{array}{l}\text { Day of life } \\
\text { (DOL) }\end{array}$ & Saline/RA & \multicolumn{1}{c}{ Saline/O } & LPS/RA & \multicolumn{1}{c}{ LPS/O } \\
\hline DOL 1 (birth) & $1.34 \mathrm{~g} \pm 0.02$ & $\mathrm{~N} / \mathrm{A}$ & $1.32 \mathrm{~g} \pm 0.01$ & $\mathrm{~N} / \mathrm{A}$ \\
DOL 5 & $3.12 \mathrm{~g} \pm 0.12$ & $2.50^{*} \mathrm{~g} \pm 0.03$ & $2.72 \mathrm{~g} \pm 0.07$ & $2.25^{*} \mathrm{~g} \pm 0.04$ \\
DOL 14 & $6.63 \mathrm{~g} \pm 0.13$ & $6.60 \mathrm{~g} \pm 0.17$ & $6.80 \mathrm{~g} \pm 0.09$ & $6.44 \mathrm{~g} \pm 0.15$ \\
DOL 21 & $9.38 \mathrm{~g} \pm 0.24$ & $8.71 \mathrm{~g} \pm 0.27$ & $8.33^{*} \mathrm{~g} \pm 0.23$ & $8.51^{*} \mathrm{~g} \pm 0.30$
\end{tabular}

Data are presented as mean \pm SE ( $n=11-12$ dams and 8-46 pups/group).

* Signifies significant different from saline/RA, $P<0.05$.

a

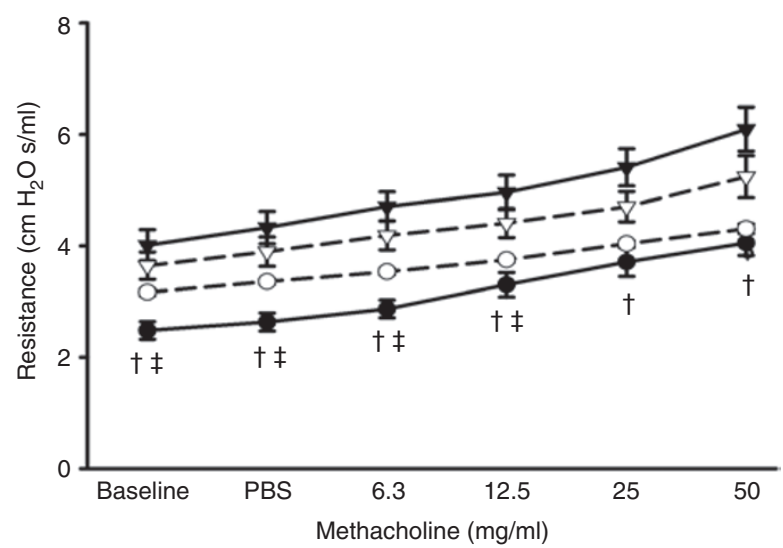

b

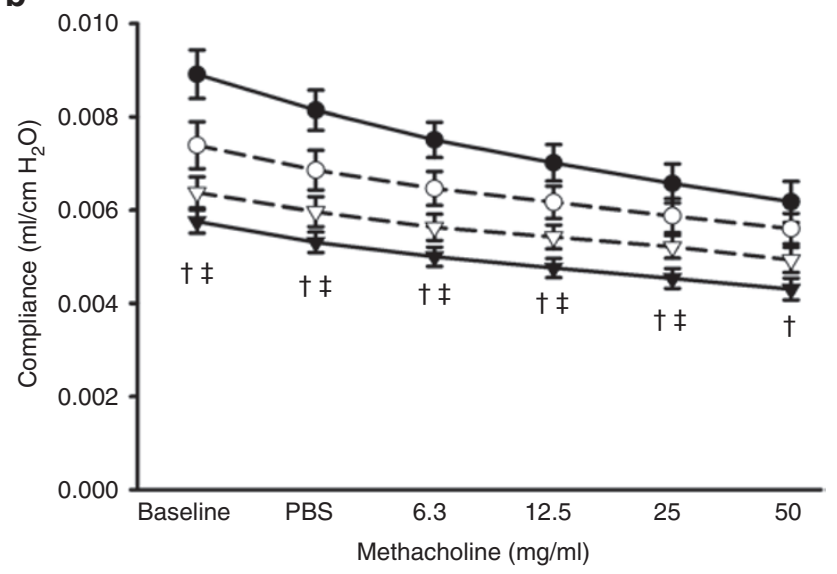

Figure 1. Effects of lipopolysaccharide (LPS) and/or $50 \% \mathrm{O}_{2}$ on pulmonary function testing at day of life 21. Black circles represent Saline/RA, white circles Saline $/ \mathrm{O}_{2}$, black triangles LPS/RA, and white triangles $\mathrm{LPS} / \mathrm{O}_{2}$. Compared to Saline/RA mice, LPS/RA mice all demonstrated increased total pulmonary resistance (denotes entire respiratory system including: airway, lungs, and chest wall) at baseline and with increasing concentrations of methacholine (a). LPS $/ \mathrm{O}_{2}$ mice had increased baseline pulmonary resistance and with methacholine up to $12.5 \mathrm{mg} / \mathrm{ml}$ (a). LPS/RA mice had decreased pulmonary compliance, while LPS/O, exposed pups had decreased compliance at baseline, with PBS, and up to $25 \mathrm{mg} / \mathrm{ml}$ methacholine (b). Data are presented as mean \pm SE ( $n=7-9$ pups/group). 'tindicates significant difference between Saline/RA and LPS/RA and ${ }^{\ddagger}$ indicates significant difference between Saline/ $\mathrm{RA}$ and $\mathrm{LPS} / \mathrm{O}_{2^{\prime}} P<0.05$.

$P<0.05)$. Furthermore, LPS/RA pups had significantly increased total airway resistance and decreased dynamic compliance in response to increasing concentrations of methacholine (Figure $1 ; P<0.05$ ). In comparison to Saline/RA, LPS/ 
$\mathrm{O}_{2}$ pups had increased resistance in response to methacholine up to $12.5 \mathrm{mg} / \mathrm{ml}$ methacholine. $\mathrm{LPS} / \mathrm{O}_{2}$-exposed pups had significantly decreased compliance in response to methacholine up to $25 \mathrm{mg} / \mathrm{ml}$ (Figure 1, panel b; $P<0.05$ ).

At baseline, the inspiratory capacity (IC) was significantly decreased in LPS/RA pups (Table 2). Tissue damping (G) is a measure related to tissue resistance and correlates to energy dissipation in the alveoli. Tissue elasticity/impedance $(\mathrm{H})$ reflects the energy conversation in the alveoli. We found that intraperitoneal LPS exposure significantly increased both tissue damping and elasticity in contrast to control

Table 2. Respiratory mechanics

\begin{tabular}{|c|c|c|c|c|}
\hline & $\begin{array}{l}\mathrm{IC} \\
(\mathrm{ml})\end{array}$ & $\begin{array}{c}\mathrm{R}_{\mathrm{n}} \\
\left(\mathrm{cmH}_{2} \mathrm{O} . \mathrm{s} / \mathrm{ml}\right)\end{array}$ & $\begin{array}{c}\mathrm{G} \\
\left(\mathrm{cmH}_{2} \mathrm{O} / \mathrm{ml}\right)\end{array}$ & $\begin{array}{c}\mathrm{H} \\
\left(\mathrm{cmH}_{2} \mathrm{O} / \mathrm{ml}\right)\end{array}$ \\
\hline Saline/RA & $0.24 \pm 0.02$ & $0.73 \pm 0.05$ & $19.37 \pm 1.01$ & $109.48 \pm 7.37$ \\
\hline Saline $/ \mathrm{O}_{2}$ & $0.18 \pm 0.02$ & $1.07^{*} \pm 0.12$ & $21.95 \pm 1.87$ & $137.42 \pm 11.58$ \\
\hline LPS/RA & $0.17^{*} \pm 0.02$ & $0.79 \pm 0.24$ & $27.91^{*} \pm 1.31$ & $167.06^{*} \pm 8.10$ \\
\hline $\mathrm{LPS} / \mathrm{O}_{2}$ & $0.18 \pm 0.01$ & $1.08^{*} \pm 0.10$ & $25.80^{*} \pm 1.34$ & $153.68^{*} \pm 9.09$ \\
\hline
\end{tabular}

Neonatal inspiratory capacity $(I C)$, airway resistance $\left(R_{n}\right.$; specific to airway resistance without lung and chest wall components), tissue damping (G), and tissue elasticity $(H)$ at baseline on DOL 21.

LPS, lipopolysaccharide.

* indicates significant different from Saline/RA, $P<0.05$. Data are presented as mean \pm SE ( $n=7-9$ pups/group).
(Table 2). Specific airway resistance $\left(\mathrm{R}_{\mathrm{n}}\right)$, correlating to the airway itself without the contributions of lung and chest wall, was significantly higher in hyperoxia-exposed pups at baseline (Table 2).

\section{Histology and Lung Morphometry}

Using standard lung morphometric techniques, the principal components of the airway were quantified to determine ASM and bronchial airway epithelial layer thicknesses as compared to total airway dimensions. Examination of $\mathrm{H} \& \mathrm{E}$ sections at DOL 14 showed no differences between the treatment groups with respect to ASM/total area (Figure 2, panels a and c). Maternal LPS, in conjunction with postnatal hyperoxia exposure resulted in greater airway epithelial thickness at DOL 14 (Figure 2, panels b and c; $P<0.05$ ).

Analysis of $\mathrm{H} \& \mathrm{E}$ sections from the four groups at DOL 21 found that LPS/RA, LPS/O ${ }_{2}$, and Saline $/ \mathrm{O}_{2}$ mice all had increased ASM/total area thicknesses (Figure 2, panels $\mathrm{d}$ and f; $P<0.05$ for each comparison). The combination of maternal LPS with postnatal hyperoxia did not result in significantly greater ASM thickening compared to LPS or hyperoxia alone (Figure 2, panels d and f; $P<0.05$ ). Unlike the findings at DOL 14 , the airway epithelial area/total area did not differ between treatment groups at DOL 21 (Figure 2, panel e). a

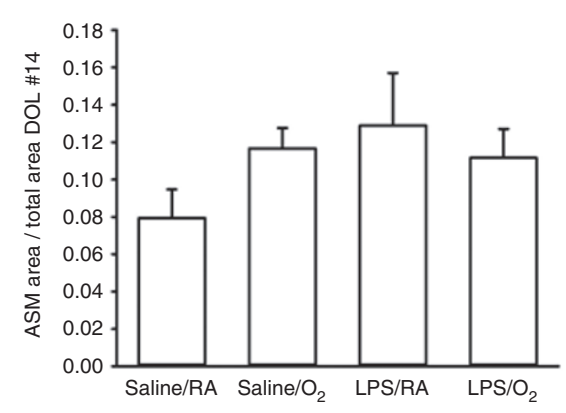

d

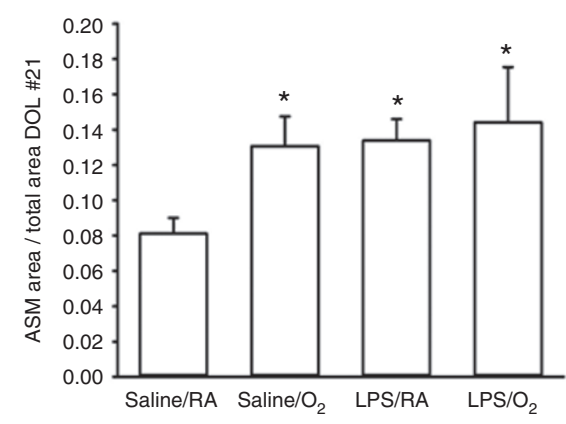

b

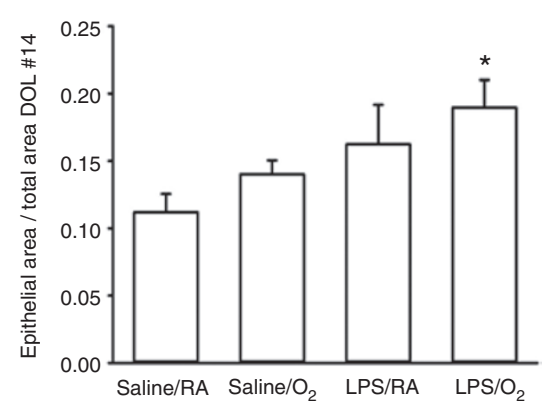

e

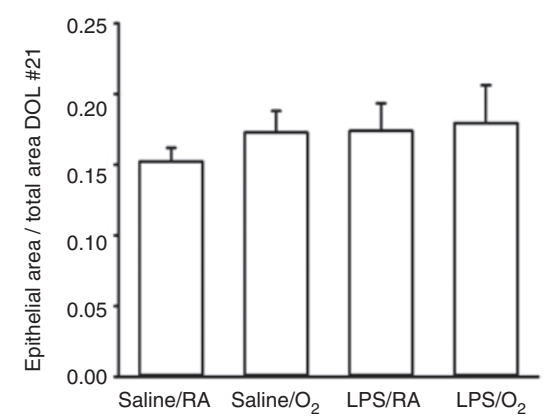

C

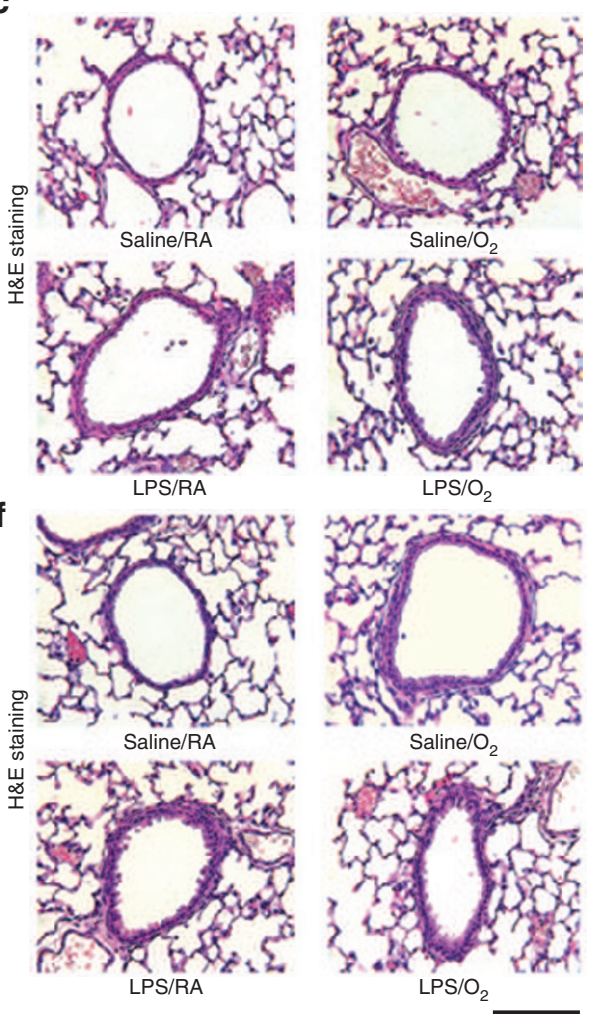

Figure 2. Lung histology and airway morphometric analyses. On DOL 14, there were no differences observed in airway smooth muscle area (a), whereas the airway epithelial area was greater at DOL 14 in LPS/O -exposed pups in comparison to Saline/RA (b). All treatment groups had increased airway smooth muscle area compared to Saline/RA on DOL 21 (d). There were no differences observed in airway epithelial area at DOL 21 between treatment groups (e). Representative images of airways stained with hematoxylin and eosin were taken at 400x magnification for each treatment at DOL 14 (c) and 21 (f) respectively. Scale bar represents $100 \mu \mathrm{m}$. Data are presented as mean \pm SE ( $n=4-6$ pups/group). * indicates significant difference from Saline/ RA, $P<0.05$. 


\section{Articles $\mid$ Faksh et al.}

a

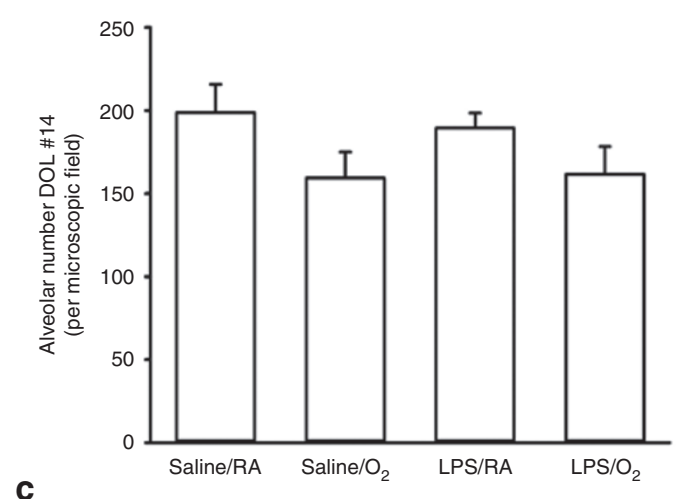

C

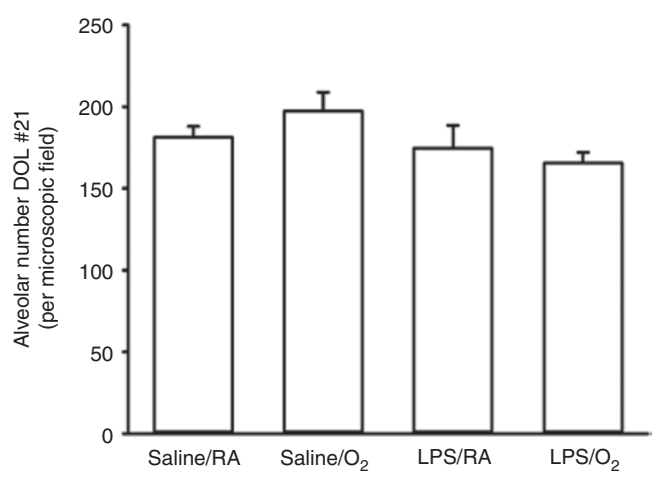

b
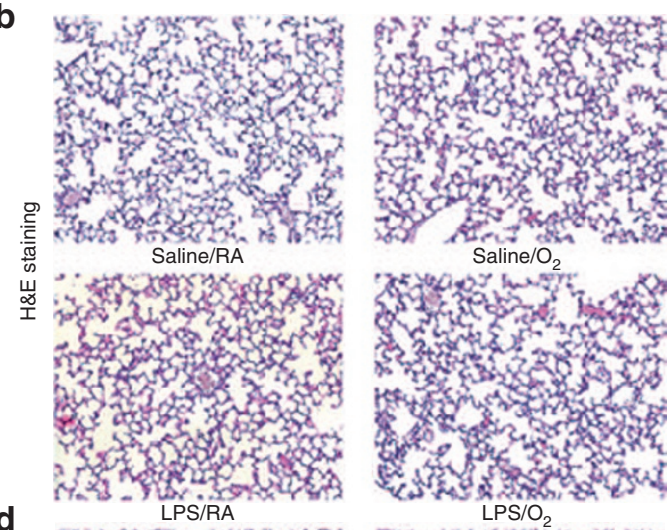

d
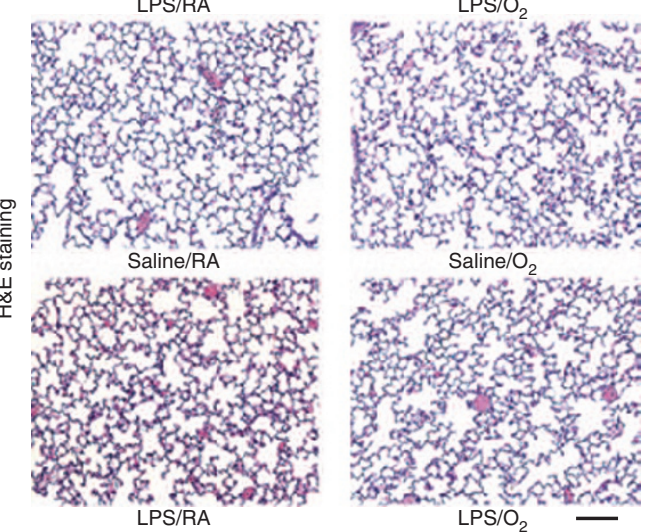

Figure 3. Lung histology and alveolar morphometric analyses. No differences were observed in alveolar number at either DOL 14 (a) or 21 (c). Representative photomicrographs of alveoli stained with hematoxylin and eosin for each treatment group at DOL 14 (b) and DOL 21 (d) were taken at $200 \times$ magnification. Scale bar represents $100 \mu \mathrm{m}$. Data are presented as mean $\pm \mathrm{SE}$ ( $n=3-6 \mathrm{pups} / \mathrm{group})$.

Alveolarization was assessed and DOL 14 and 21. Neither intraperitoneal LPS administration nor postnatal hyperoxia exposure had any effect on alveolar appearance in our model. There were no changes observed in alveolar number between treatment groups (Figure 3). Moreover, we did not observe any differences in alveolar area between treatment groups at either DOL 14 or 21 (data not shown).

\section{Collagen Deposition, Elastin Staining, and $\alpha$-Smooth Muscle Actin Immunostaining}

Airway collagen deposition was qualitatively examined in Masson's trichrome-stained lung tissue sections at DOL 14 and 21 (Figure 4, panels a and c). Saline/O $\mathrm{O}_{2}, \mathrm{LPS} / \mathrm{RA}$, and LPS/O $\mathrm{O}_{2}^{-}$ exposed pups all showed more collagen within the ASM layer at both postnatal time points compared to Saline/RA mice (Figure 4, panels a and c). Similarly, elastin deposition was qualitatively examined in Accustain elastic-stained lung tissue sections at DOL 14 and 21 (Figure 4, panels $\mathrm{b}$ and d). There were no differences observed in elastin staining among the different treatment groups. On DOL 14 and 21, Saline/O $\mathrm{O}_{2}$, LPS/ $\mathrm{RA}$, and LPS $/ \mathrm{O}_{2}$ demonstrated a relative increased qualitative airway $\alpha$-smooth muscle actin expression around the airway as compared to Saline/RA (Figure 5). However, only on DOL 14 was the airway $\alpha$-smooth muscle actin expression significantly increased quantitatively amongst the treatment groups in relation to Saline/RA, control (Figure 5, panel a). Under low magnification, we did not observe any changes in collagen, elastin, or $\alpha$-smooth muscle actin expression in the distal lung parenchyma (data not shown).

\section{Proinflammatory Cytokine and Receptor Expression}

In lung tissues derived from mice at birth, mRNA levels of inflammatory genes were assessed to determine the contribution of maternal inflammation on the neonate. Maternally derived proinflammatory mediators have been implicated in the fetal inflammatory response reaction and in the pathogenesis of lung diseases. Compared to saline controls, maternal exposure to LPS also significantly increased TLR4, IL-6, IL-1 $\beta$, and TNF $\alpha$ mRNA levels in lung tissues of newborn pups (Figure 6, panel a; $P<0.05$ ). At DOL 1 , mRNA level of TGF- $\beta$, a profibrotic marker, was significantly higher in pups of LPSexposed mothers (Figure 6, panel a; $P<0.05$ ), however there was no change observed in $C T G F$, a remodeling gene downstream of TGF $\beta$. With the exception of $I L-1 \beta$ which was significantly increased in the LPS/RA treatment group, there were no differences observed at DOL 5 with respect to proinflammatory or profibrotic mediators (Figure 6, panel b).

\section{ASM Expression of Remodeling Genes}

Laser capture microdissection (LCM) was utilized to isolate the ASM layer so as to analyze mRNA expression of mediators that modulate airway remodeling over time on postnatal DOL 5,14 , and 21. This approach permitted quantification of ECM changes that could not be performed using the qualitative 

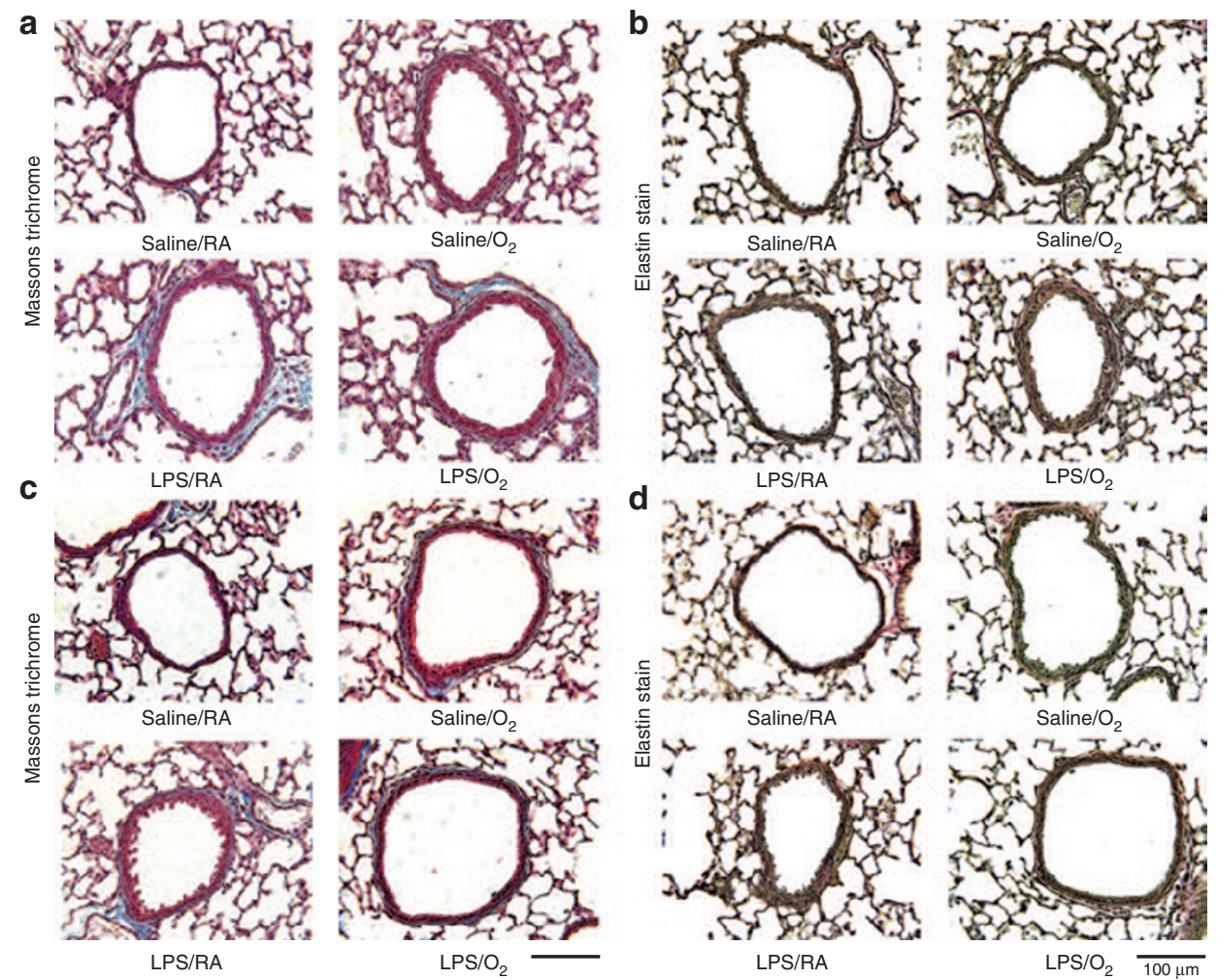

Figure 4. Collagen and elastin deposition in the neonatal airway. Airway collagen deposition was qualitatively examined in Masson's trichrome-stained lung tissue sections at DOL 14 (a) and DOL 21 (c). Compared to Saline/RA mice, Saline/O ${ }_{2}$ lipopolysaccharide (LPS)/RA, and LPS/O ${ }_{2}$ demonstrated greater blue staining at day 14 (a) and 21 (c). Collagen deposition in blood vessels served as a positive control in Saline/RA mice. Airway elastin deposition was qualitatively examined in lung sections using an Accustain kit at DOL 14 (b) and DOL 21 (d). There were no differences observed in elastin staining between the different treatment groups. Representative images were taken at $400 \times$ magnification. Scale bar represents $100 \mu \mathrm{m}$.

trichome or other stains. Early in neonatal life, on DOL 5, no significant differences were observed between treatment groups for Collagen-1, Collagen-3, TGF $\beta$, or CTGF mRNA levels (Figure 7). Although at DOL 14, there were no significant changes in the treatment groups for Collagen-1 mRNA (Figure 7, panel a), we observed higher Collagen-3 mRNA levels in Saline $/ \mathrm{O}_{2}$ and $\mathrm{LPS} / \mathrm{O}_{2}$-exposed pups (Figure 7, panel b). Similarly at DOL 21, there were no differences observed for Collagen-1 mRNA, however LPS/RA-exposed pups demonstrated significantly increased mRNA expression of Collagen-3 (Figure 7, panel b; $P<0.05$ ). TGF $\beta$ was not significantly upregulated until DOL 21 in the treatment groups (Figure 7, panel c). Downstream of TGF $\beta, C T G F$ can promote a fibrotic response by stimulating extracellular matrix synthesis $(17,24,25)$. At DOL 14, CTGF was found to be significantly decreased in LPS-exposed pups, however an increase in CTGF was later observed in all treatment groups on DOL 21 (Figure 7, panel d).

\section{DISCUSSION}

Recent studies demonstrate that there is an association between antenatal maternal inflammation, subsequent preterm birth, and development of recurrent wheeze and asthma $(11,12,26)$. However, the pathophysiological relationship between inflammation and development of bronchial airway disease remains poorly understood. Furthermore, the mechanisms contributing to airway remodeling and dysfunction in preterm infants remain undefined. Using a model of systemic maternal inflammation and newborn hyperoxia exposure, we assessed the effect of maternal inflammation on airway structure and function. We hypothesized that compared to Saline/ RA exposed pups, LPS/hyperoxia $\left(50 \% \mathrm{O}_{2}\right)$ exposed pups will demonstrate enhanced airway reactivity and remodeling.

Exposure to antenatal inflammation and hyperoxia in rodent models augments airway contractility and structure in newborn pups (14-16). At baseline and during methacholine challenge, LPS/RA and LPS/O ${ }_{2}$ pups exhibited higher resistance and lower compliance than Saline/RA pups. Tissue damping and elasticity, which reflect lung stiffening, were also increased in both LPS-exposed groups. While resistance and compliance of Saline/ $\mathrm{O}_{2}$ pups were not significantly different from Saline/ RA pups, they did have increased specific airway resistance $\left(\mathrm{R}_{\mathrm{n}}\right)$. These data suggest that maternal LPS exposure resulted in stiffer lungs, which is in contrast to exposure to only $50 \%$ $\mathrm{O}_{2}$ which had modest effects on the lung. However, in contrast to our hypothesis, hyperoxia exposure did not amplify the detrimental effects of LPS on lung structure and function. These findings are in contrast to previous studies in a rat chorioamnionitis model in which administration of intra-amniotic LPS alone did not alter airway function but did enhance the effects of $60 \% \mathrm{O}_{2}(14)$. We speculate that the differences between models could be due to differences in LPS administration, 


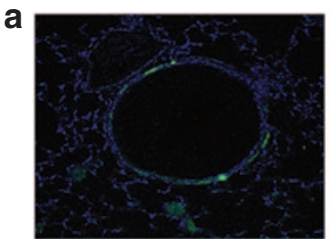

Saline/RA

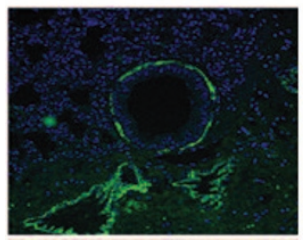

LPS/RA

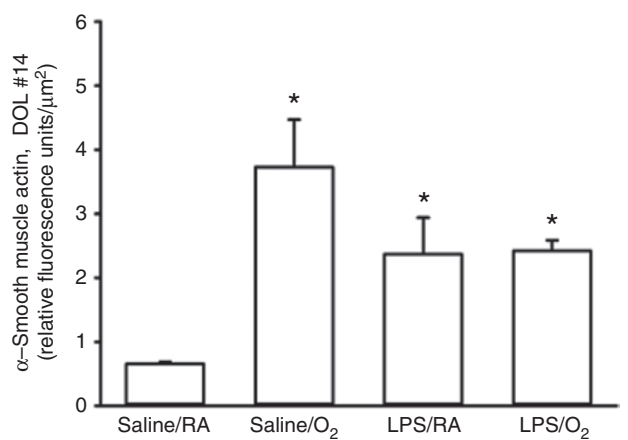

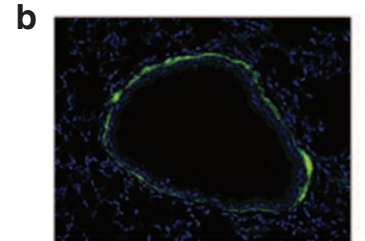

Saline/RA

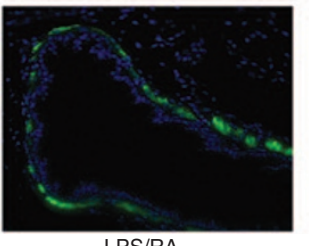

LPS/RA

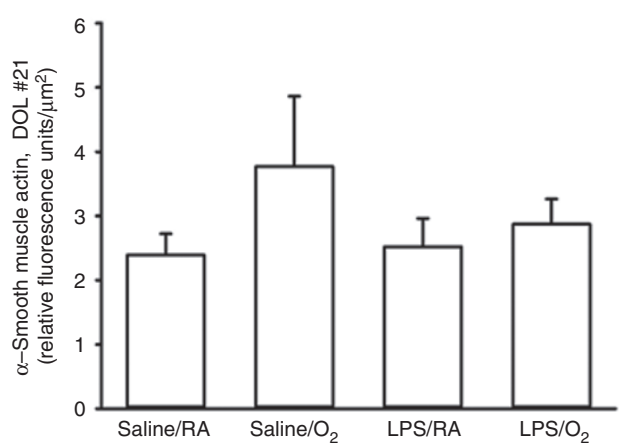

Figure 5. $\alpha$-Smooth muscle actin immunohistochemistry in the neonatal airway. There was significantly increased $\alpha$-smooth muscle actin fluorescence visualized around the airways of hyperoxia and lipopolysaccharide (LPS)-exposed pups at postnatal DOL 14, as compared to Saline/RA control pups (a). At postnatal DOL 21, no significant differences were observed (b). Representative images were taken at 400× magnification. Fluorescence was normalized to airway area. Scale bar represents $100 \mu \mathrm{m}$. Data are presented as mean \pm SE ( $n=3-6$ pups/group). * indicates significant difference from Saline/ RA, $P<0.05$.

concentration or duration of hyperoxia exposure, or species. Furthermore, these differences emphasize the need for consideration of the model per se in interpretation of perinatal inflammation or oxygen effects on the developing lung.

Diminished lung function in preterm infants and young children with recurrent wheeze and asthma is accompanied by structural airway changes (27). Remodeling of the airway is characterized by increased thickening within the airway smooth muscle layer and deposition of the ECM (28). Compared to controls, Saline/O, ${ }_{2}$ LPS/RA, and LPS/O ${ }_{2}$ pups exhibited thicker airways which can be attributed primarily to thickening within the ASM layer. However, these observations were not observed until DOL 21 even though hyperoxia exposure occurred only for the first $7 \mathrm{~d}$, suggesting that perinatal insults have long term effects on airway structure. Exposed pups also exhibited increased collagen deposition on DOL 14 and 21 , which contributes to airway stiffening. No changes in elastin deposition were demonstrated amongst the treatment groups at DOL 14 and 21, suggesting that imbalances in airway collagen may represent more of a primary contributing factor to alterations in airway function.

While higher levels of hyperoxia are known to disrupt alveolar development $(14,15,29)$, we did not observe alveolar simplification, alterations in either alveolar $\alpha$-smooth muscle actin, elastin, or collagen deposition amongst our treatment groups. This is an important distinction between our study and previous exploration of the toxicity of hyperoxia in the context of bronchopulmonary dysplasia $(14,15,29)$. We propose that moderate level of hyperoxia exposure results in more prominent (detrimental) changes in proximal (conducting) airways and thus places the developing airway at risk of associated diseases such as wheezing and asthma, rather than bronchopulmonary dysplasia.

Antenatal LPS exposure heightens expression of proinflammatory cytokines in the newborn mouse lung $(2,5,9,15,21)$. Maternally derived inflammatory mediators, instigated by LPS administration, promptly traverse the placenta and are transmitted to the fetus (30). Maternal inflammation results in increased expression of proinflammatory cytokines within the amniotic fluid and commonly leads to preterm delivery (5). These events perpetuate a proinflammatory response in the fetal lung, which may be further enhanced by hyperoxia exposure. In our study, maternal LPS exposure significantly increased mRNA expression of multiple key proinflammatory mediators: $I L-6, I L-1 \beta, T N F \alpha$, TLR4, and TGF $\beta$ at DOL 1. At DOL 5 , the majority of these inflammatory changes had returned to baseline levels. These data suggest that systemic maternal inflammation creates a transient inflammatory milieu in the newborn lung which could contribute to the observed altered airway structure later in life.

A limitation of our immunohistochemical approaches to evaluating changes in airway structure is that they are not 

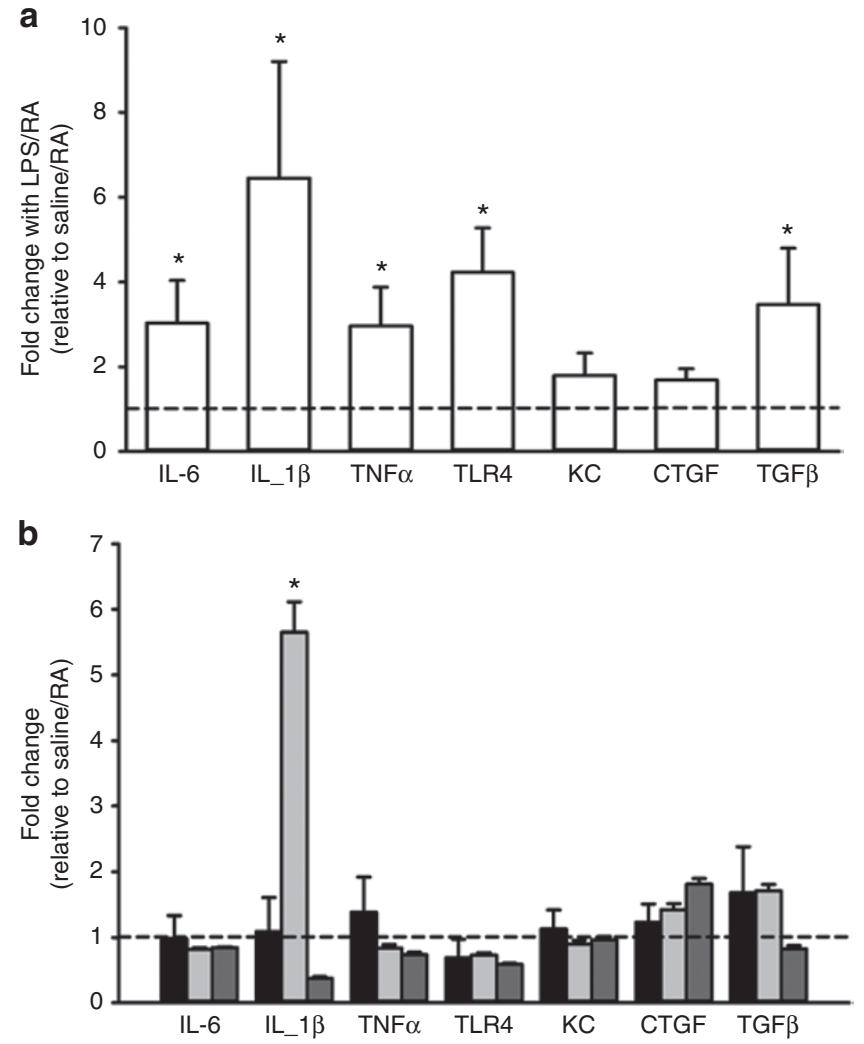

Figure 6. Proinflammatory cytokine mRNA levels in neonatal lung. IL-6, IL-1 $\beta, T N F-\alpha, T L R 4, K C$, connective tissue growth factor (CTGF), and TGF $\beta$ mRNA expression were measured in lung homogenates in pups born to saline- or lipopolysaccharide (LPS)-injected dams at day 1. LPS significantly increases IL-6, IL- $1 \beta, T N F-\alpha, T L R 4$, and TGF $\beta$ (a). At postnatal DOL 5, these changes were not as pronounced in the treatment groups (black bars representing Saline/ $\mathrm{O}_{2}$, light gray LPS/RA, and dark gray $\mathrm{LPS} / \mathrm{O}_{2}$ with hash tag line corresponding to Saline/RA control), without significant changes observed other than IL-1 $\beta$ for the LPS/RA-exposed pups (b). Data are presented as mean $\pm \mathrm{SE}$ ( $n=7$ pups/group at DOL 1, $n=3-6$ pups/ group at DOL 5). * indicates significant difference from saline or Saline/RA control, $P<0.05$.

quantitative. Using LCM, we were able to quantify mRNA levels of genes relevant to airway remodeling and the ECM within the airway smooth muscle layer per se at different postnatal time points. This is the first study to our knowledge which explores the impact of perinatal inflammation and subsequent postnatal hyperoxia exposure within the airway smooth muscle over the early postnatal period. We found increased mRNA expression of collagen-3 as compared to collagen-1 at DOL 14 and 21 . We speculate that our finding of increased collagen-3 and decreased collagen-1 may be due to differential localization of collagen-1 vs. collagen-3 in the airway smooth muscle vs. airway epithelial layer. Future investigations are needed to distinguish between changes in ECM protein expression in the airway smooth muscle vs. airway epithelial layer and their relative contributions to the remodeled airway. Another factor which may play a role in our findings relates to timing. Remodeling in the airway smooth muscle may result in increased collagen-3 earlier, followed by alterations in collagen-1 at later time points, possibly beyond day of life 21 . Furthermore, alterations in ECM proteins represent a balance between generation of new protein and degradation (e.g., mediated via proteases within the matrix). These areas are topics for future studies.

In the present study, we explored potential mechanisms underlying hyperoxia and/or LPS effects on the airway. In this regard, we found changes in TGF $\beta$ and CTGF which could be potential mechanisms, although we did not specifically explore their roles. Our findings are consistent with previous studies which demonstrate that systemic maternal inflammation and hyperoxia increase TGF $\beta$ and collagen expression in the postnatal lung (15). TGF $\beta$ promotes profibrotic responses through enhancing ECM deposition, cell proliferation, and secretion of growth factors such as CTGF $(17,24,25,31,32)$. CTGF was found in prior studies to be increased in the lungs of newborn pups exposed to hyperoxia where it is thought to contribute to collagen deposition in the neonatal lung $(33,34)$. The effects of TGF $\beta$ on the airway during neonatal hyperoxia are less known, but in adult ASM TGF $\beta$ increases proliferation, collagen deposition, and CTGF secretion $(24,25,35)$. Taken together, we postulate that early activation of proinflammatory cytokines, including TGF $\beta$, could contribute to the altered structural and functional changes to the airway in LPS and hyperoxia exposed pups. Here, we noted that at DOL 14, CTGF expression decreased in LPS-exposed pups, although collagen levels were increased. It is possible that CTGF plays a role in modulating collagens or other ECM proteins at certain time periods following the initial insult, but TGF $\beta$ may induce other factors that also influence ECM proteins: topics that remain to be explored. This concept is supported by a prior study which found that CTGF production is initially reduced in endotoxin-exposed ewes (17). Furthermore, the mechanisms by which TGF $\beta$ exerts its effects in developing airway remain to be determined. For example, we did not observe changes in Smad phosphorylation, but it is possible that alternative mechanisms related to TGF $\beta$ signaling, such as MAP kinases, are involved.

Overall, our data suggest that LPS and hyperoxia upregulate proremodeling mechanisms within the ASM layer which can contribute to altered airway structure. These novel studies demonstrate that maternal LPS had pronounced effects on airway structure and function. We observed that antenatal LPS exposure increased ASM expression of growth factors, TGF $\beta$ and CTGF, which are known to promote airway remodeling. Although hyperoxia exposure had an effect on airway structure, the effects of hyperoxia on airway function were less pronounced. In addition, TGF $\beta$ and CTGF expression in hyperoxia-exposed pups was similar to maternal LPS-exposed pups. These observations suggest that LPS may separately modulate other factors that could enhance ASM contractility. Additionally, hyperoxia did not synergistically augment the effects of maternal LPS on the airway. We postulate that use of moderate hyperoxia levels during respiratory support for preterm infants does not further exacerbate the effects of maternal inflammation. The effects of antenatal inflammation on airway function are not well understood, thus exploring the contributing mechanisms could 
a

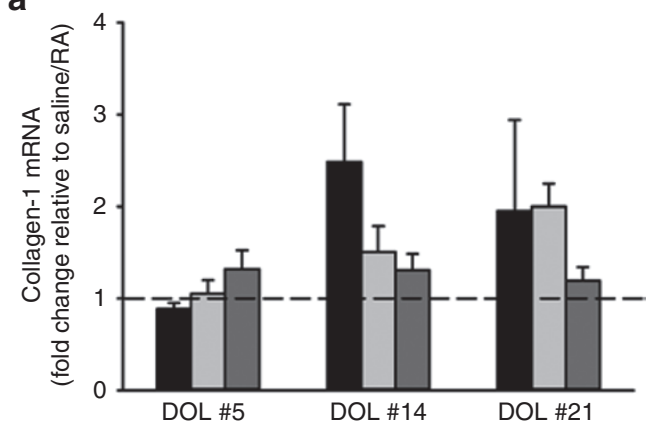

C

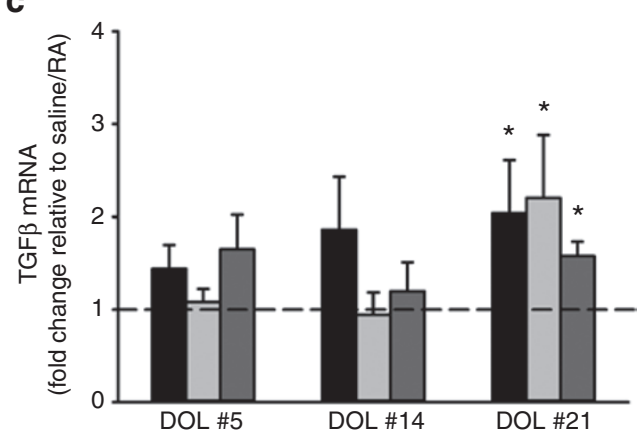

b

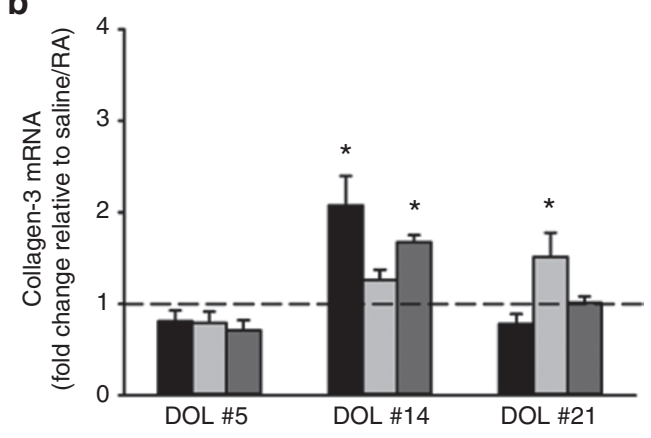

d

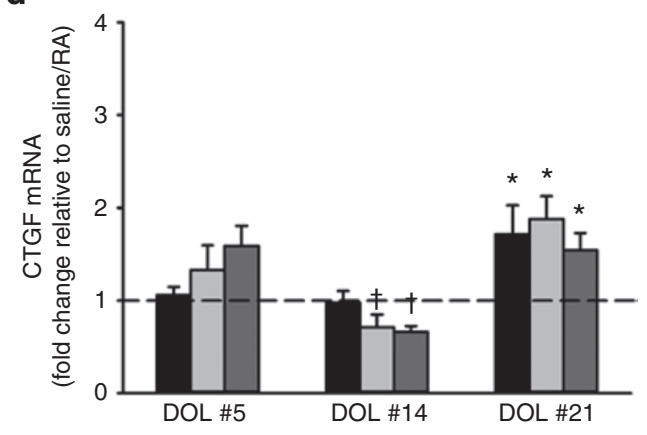

Figure 7. Changes in neonatal airway remodeling during lipopolysaccharide (LPS) and hyperoxia exposure. Laser capture micro-dissection was performed on frozen lung sections. Expression of Collagen-1, Collagen-3, TGF $\beta$, and connective tissue growth factor (CTGF) was analyzed by quantitative real-time PCR at postnatal DOL 5, 14, and 21. Black bars represent Saline/ $\mathrm{O}_{2}$, light gray LPS/RA, and dark gray LPS/O with hash tag line corresponding to Saline/RA control. No statistically significant increases were observed in Collagen-1 mRNA among the treatment groups at any postnatal day (a). At DOL 14, there was a statistically significant increase in Collagen-3 for Saline/O and LPS/O -exposed pups, and then at DOL 21 for LPS/RA-exposed pups (b). At DOL 5 and DOL 14, no changes in TGF $\beta$ were observed between the treatment groups (c). Later on DOL 21 all treatment groups had significant increase in TGF $\beta$ mRNA (c). CTGF mRNA levels were lower among LPS-exposed mice on DOL 14 as compared to Saline/RA controls. Increased CTGF mRNA expression was notable at DOL 21 with significant changes in all treatment groups (d). Data are presented as mean \pm SE $(n=3-6$ pups/ group at DOL 5, $n=5-9$ pups/group at DOL 14, and $n=4$ pups/group at DOL 21). ${ }^{*}$ indicates significant difference from Saline/RA control; ${ }^{+}$indicates significant decrease from Saline/RA control, $P<0.05$.

help identify potential therapeutic strategies to improve airway function of preterm infants exposed to antenatal inflammation.

\section{METHODS}

\section{Animals and Exposure}

All animal study protocols were approved by the Mayo Clinic Institutional Animal Care and Use Committee and conducted in accordance to guidelines derived from the National Institutes of Health Guide for the Care and Use of Laboratory Animals. C57/Bl6 (Jackson Laboratories, Bar Harbor, ME) female mice were housed for at least 1 week prior to breeding. Observation of a vaginal plug was designated as embryonic day 1 . On embryonic day 16 (E16), pregnant dams were injected intraperitoneally (IP) with $100 \mu \mathrm{l}$ sterile saline or LPS $(200 \mu \mathrm{g} /$ $\mathrm{kg}$, LPS from Escherichia coli 055:B5, L4524; Sigma Aldrich, St. Louis, MO). LPS dose was selected based on pilot studies to determine an effective dose that resulted in a viable litter. Each litter of newborn mice was paired with a litter born to a dam receiving the same E16 treatment (LPS or saline), and the pups were pooled and redistributed randomly. Within $16 \mathrm{~h}$ of birth, one of the paired groups of pups was exposed to room air (RA) or hyperoxia $\left(50 \% \mathrm{O}_{2}\right)$ for $7 \mathrm{~d}$. Oxygen levels were continuously monitored using an oxygen sensor. Nursing dams were rotated between their RA and O litter every $24 \mathrm{~h}$ to circumvent oxygen toxicity. On postnatal day of life (DOL) 7, all pups were returned to RA. Pups were sacrificed on DOL 1 (birth), 5, 14, and 21 and lung tissue collected. Lungs in each litter were randomly selected to be either immediately frozen and stored at $-80^{\circ} \mathrm{C}$ for future use or inflated to $25 \mathrm{~cm} \mathrm{H}_{2} \mathrm{O}$ pressure with $4 \%$ paraformaldehyde.

\section{Airway Mechanics}

The FlexiVent (SCIREQ, Montreal, Canada) was utilized at postnatal DOL 21 (36). Mice were anesthetized with IP ketamine $(20 \mathrm{mg} /$ $\mathrm{kg})$ and xylazine $(2 \mathrm{mg} / \mathrm{kg})$, placed in a supine position, the trachea was cannulated with a 20 -gauge blunt Luer cannula, and the animals ventilated with positive pressure. After baseline total lung resistance $\left(\mathrm{R}_{\mathrm{L}}\right)$ and compliance $(\mathrm{C})$ was assessed, responses to increasing doses of nebulized methacholine (PBS (0), 6.3, 12.5, 25, and $50 \mathrm{mg} / \mathrm{ml}$ )) were measured (36). Additional measurements undertaken at baseline include: specific airway resistance $\left(R_{n}\right)$, tissue damping $(G)$, and tissue elasticity/impedance $(H)$. Total resistance $\left(R_{I}\right)$ is distinct from specific airway resistance $\left(R_{n}\right)$ in that the former accounts for airway, lungs, and chest wall, whereas the latter accounts for airway alone (36).

\section{Histology and Morphometric Evaluation}

At postnatal DOL 14 and 21, animals were sacrificed and lungs were inflated with $4 \%$ paraformaldehyde at a pressure of $25 \mathrm{~cm} \mathrm{H} O$, and subsequently paraffin embedded, sectioned at $10 \mu \mathrm{m}$, and taken through standard protocols for hematoxylin and eosin (H\&E), Masson trichrome (collagen), and Accustain (elastin) staining (Sigma Aldrich).

For all four treatment groups (Saline/RA, Saline/O, LPS/RA, and LPS $/ \mathrm{O}_{2}$ ) at DOL 14 and 21, H\&E-stained lung cross-sections were examined under light microscopy at $400 \times$ magnification to assess airway and alveolar structure using previously described techniques (37-39). Using ImageJ software (National Institutes of Health; http:// rsb.info.nih.gov/ij), airway epithelial layer, ASM layer, and total airway thicknesses were quantified using the circular method (37). All of the morphometric and image analysis measurements were performed 
Table 3. Mouse primers used for quantitative real-time PCR

\begin{tabular}{|c|c|c|}
\hline Gene & Forward primer & Reverse primer \\
\hline S16 & TGCAGGTCTTCGGACGCAAGAAAA & CGAATATCCACACCAGCAAATCGC \\
\hline IL-6 & TGGGGCTCTTCAAAAGCTCC & AGGAACTATCACCGGATCTTCAA \\
\hline$T N F-\alpha$ & GGGTCGCACCATGAAGGAG & GAAGTGGTAGTGGTAGCTTTCG \\
\hline TLR4 & ACACCTGGATAAATCCAGCCACTGA & GCTTGAATCCCTGCATAGAGGTAG \\
\hline Connective tissue growth factor & GACCCAACTATGATGCGAGCC & CCCATCCCACAGGTCTTAGAAC \\
\hline TGF- $\beta 1$ & AGACCACATCAGCATTGAGTG & GGTGGCAACGAATGTAGCTGT \\
\hline Collagen-1 & GAAGTCAGCTGCATACACAATGGCCT & GATACGTATTCTTCCGGGCAGAAAGC \\
\hline Collagen-3 & CTGTAACATGGAAACTGGGGAAA & CCATAGCTGAACTGAAAACCACC \\
\hline E-Cadherin & AACAGGCCAGAGTTTACCCAGGAGG & GGTGTAGGCGATGGCAGCGTT \\
\hline
\end{tabular}

by a blinded investigator. Three distinct airways per section using at least three sections per animal were randomly sampled. An average was calculated from at least four different mice per treatment. Alveolarization was also assessed using ImageJ software at DOL 14 and 21. Five fields of alveoli per mouse, with at least four mice per treatment, at 200× magnification were randomly selected. Alveolar number and area were calculated using standard techniques (39).

\section{Immunohistochemistry}

Cross-sections $(10 \mu \mathrm{m})$ from paraffin embedded lungs from each treatment group at postnatal DOL 14 and 21 were deparaffinated, rehydrated, and prepared for immunostaining. Mouse lung cross-sections were blocked in $10 \%$ normal donkey serum with $0.3 \%$ Triton trisbuffered saline (TBS) for $30 \mathrm{~min}$, and incubated overnight in $4{ }^{\circ} \mathrm{C}$ in TBS only (unstained control) or $1 \mu \mathrm{g} / \mathrm{ml}$ of monoclonal anti- $\alpha$-smooth muscle actin (Sigma Aldrich, A2547) primary antibody overnight. Slides were then washed and incubated for $2 \mathrm{~h}$ in Alexa 488 anti-mouse secondary antibody (Invitrogen, Carlsbad, CA) using a 1:200 dilution. DAPI stain was used for nuclei visualization. Slides were imaged using an oil immersion lens on a Nikon Eclipse Ti microscope and pictures were acquired under the same conditions and immunofluorescence was subsequently quantified using Nikon NIS-Elements imaging software for each treatment group on DOL 14 and 21 respectively.

\section{Laser Capture Microdissection}

At DOL 5, 14, and 21, RNase-free frozen lung sections at $10 \mu \mathrm{m}$ were placed on prechilled clean slides, dehydrated with ethanol, and placed on a Veritas microdissection system (Arcturus, Molecular Devices, Sunnyvale, CA). Under light microscopy (200× magnification), small airways (300-350 $\mu \mathrm{m}$ diameter) were visualized and the ASM and airway epithelial layers were distinguished using software areas of interest (40). An infrared laser was used to microdissect the delineated regions onto CapSure Macro LCM caps. Total RNA (2 caps/animal) from the ASM layers were processed for quantitative real-time PCR, as previously described (40). Quantification of smooth muscle (smooth muscle actin) and epithelial markers (E-cadherin) were used to evaluate the homogeneity and relative purity of the tissue source for RNA.

\section{Quantitative Real-Time PCR}

Mouse lung tissue and frozen sections were prepared under RNasefree conditions. Total RNA was extracted from lung tissue using the RNeasy Mini Kit (Qiagen, Valencia, CA) while total RNA was isolated from LCM caps using the RNeasy micro kit (Qiagen). Using the transcriptor reverse transcription kit (Roche, Indianapolis, IN) complementary (cDNA) was synthesized. Real-time PCR was performed using LC480 LightCycler (ABI, Carlsbad, CA). Each cDNA sample was plated in triplicate and data in each category (Saline/RA, Saline $/ \mathrm{O}_{2}$, LPS/RA, and $\mathrm{LPS} / \mathrm{O}_{2}$ ) were pooled for statistical analysis. Message RNA (mRNA) expression was calculated by the normalization of cycle threshold $(\mathrm{C}(\mathrm{t})$ ) values of target gene to reference gene (ribosomal protein S16). The relative fold change was calculated using the $\Delta \Delta \mathrm{Ct}$ method. Primers used for quantitative real-time PCR are listed in Table 3.

\section{Statistical Analysis}

A total of four to nine litters were utilized for experiments from each of the four treatment groups: Saline/RA, Saline/O, LPS/RA, and LPS/ $\mathrm{O}_{2}$. Pups from at least two litters were used for all analyses. Statistical analyses were performed using Sigma Plot (SYSTAT, San Jose, CA) software statistical package. Data were analyzed by unpaired Student's $t$-test or two-way ANOVA with Tukey post hoc analysis. Statistical significance was established at $P<0.05$. Values are means \pm SE.

\section{ACKNOWLEDGMENTS}

This paper was accepted for oral platform presentation at the American Academy of Pediatrics (Section on Perinatal Medicine) 85th Perinatal and Developmental Medicine Symposium in Fort Myers, FL on 14 November 2014. The authors would like to thank Logan Manlove, B.S. (Department of Anesthesiology, Mayo Clinic, Rochester, MN), Michelle Freeman, B.S. (Department of Anesthesiology, Mayo Clinic, Rochester, MN), and Yun-Hua Fang (Department of Anesthesiology, Mayo Clinic, Rochester, MN) for their contributions to this manuscript.

\section{STATEMENT OF FINANCIAL SUPPORT}

The authors acknowledge funding from the Mayo Clinic (Rochester, MN) Departments of Obstetrics and Gynecology (A.F.) and Anesthesiology (Y.S.P., C.M.P.), and grants from the National Institutes of Health (Bethesda, MD) T32 HL105355 (R.D.B., E.R.V.), F32 HL123075 (R.D.B.), R01 HL056470 (Y.S.P, R.J.M.), R01 HL088029 (Y.S.P.).

Disclosure: The authors report no conflict of interest.

\section{REFERENCES}

1. Bastek JA, Gómez LM, Elovitz MA. The role of inflammation and infection in preterm birth. Clin Perinatol 2011;38:385-406.

2. Kunzmann S, Collins JJ, Kuypers E, Kramer BW. Thrown off balance: the effect of antenatal inflammation on the developing lung and immune system. Am J Obstet Gynecol 2013;208:429-37.

3. Dessardo NS, Dessardo S, Mustać E, Banac S, Petrović O, Peter B. Chronic lung disease of prematurity and early childhood wheezing: is foetal inflammatory response syndrome to blame? Early Hum Dev 2014;90:493-9.

4. Britt RD Jr, Faksh A, Vogel E, Martin RJ, Pabelick CM, Prakash YS. Perinatal factors in neonatal and pediatric lung diseases. Expert Rev Respir Med 2013;7:515-31.

5. Viscardi RM. Perinatal inflammation and lung injury. Semin Fetal Neonatal Med 2012;17:30-5.

6. Kallapur SG, Presicce P, Rueda CM, Jobe AH, Chougnet CA. Fetal immune response to chorioamnionitis. Semin Reprod Med 2014;32:56-67. 


\section{Articles Faksh etal.}

7. Kemp MW, Saito M, Newnham JP, Nitsos I, Okamura K, Kallapur SG. Preterm birth, infection, and inflammation advances from the study of animal models. Reprod Sci 2010;17:619-28.

8. Kumar R, Yu Y, Story RE, et al. Prematurity, chorioamnionitis, and the development of recurrent wheezing: a prospective birth cohort study. J Allergy Clin Immunol 2008;121:878-84.e6.

9. Westover AJ, Moss TJ. Effects of intrauterine infection or inflammation on fetal lung development. Clin Exp Pharmacol Physiol 2012;39:824-30.

10. Saugstad OD, Sejersted Y, Solberg R, Wollen EJ, Bjørås M. Oxygenation of the newborn: a molecular approach. Neonatology 2012;101:315-25.

11. Collier CH, Risnes K, Norwitz ER, Bracken MB, Illuzzi JL. Maternal infection in pregnancy and risk of asthma in offspring. Matern Child Health J 2013;17:1940-50.

12. Calvani M, Alessandri C, Sopo SM, et al.; Lazio Association of Pediatric Allergology (APAL) Study Group. Infectious and uterus related complications during pregnancy and development of atopic and nonatopic asthma in children. Allergy 2004;59:99-106.

13. Hirota JA, Hackett TL, Inman MD, Knight DA. Modeling asthma in mice: what have we learned about the airway epithelium? Am J Respir Cell Mol Biol 2011;44:431-8.

14. Choi CW, Kim BI, Mason SN, Potts-Kant EN, Brahmajothi MV, Auten RL. Intra-amniotic LPS amplifies hyperoxia-induced airway hyperreactivity in neonatal rats. Pediatr Res 2013;74:11-8.

15. Velten M, Britt RD Jr, Heyob KM, et al. Prenatal inflammation exacerbates hyperoxia-induced functional and structural changes in adult mice. Am J Physiol Regul Integr Comp Physiol 2012;303:R279-90.

16. Velten M, Hutchinson KR, Gorr MW, Wold LE, Lucchesi PA, Rogers LK. Systemic maternal inflammation and neonatal hyperoxia induces remodeling and left ventricular dysfunction in mice. PLoS One 2011;6:e24544.

17. Kunzmann S, Speer CP, Jobe AH, Kramer BW. Antenatal inflammation induced TGF-beta1 but suppressed CTGF in preterm lungs. Am J Physiol Lung Cell Mol Physiol 2007;292:L223-31.

18. Tang JR, Seedorf GJ, Muehlethaler V, et al. Moderate postnatal hyperoxia accelerates lung growth and attenuates pulmonary hypertension in infant rats after exposure to intra-amniotic endotoxin. Am J Physiol Lung Cell Mol Physiol 2010;299:L735-48.

19. Burgess JK, Ceresa C, Johnson SR, et al. Tissue and matrix influences on airway smooth muscle function. Pulm Pharmacol Ther 2009;22:379-87.

20. Doherty T, Broide D. Cytokines and growth factors in airway remodeling in asthma. Curr Opin Immunol 2007;19:676-80.

21. Kunzmann S, Collins JJ, Yang Y, et al. Antenatal inflammation reduces expression of caveolin-1 and influences multiple signaling pathways in preterm fetal lungs. Am J Respir Cell Mol Biol 2011;45:969-76.

22. Makinde T, Murphy RF, Agrawal DK. The regulatory role of TGF-beta in airway remodeling in asthma. Immunol Cell Biol 2007;85:348-56.

23. Leask A, Abraham DJ. TGF-beta signaling and the fibrotic response. FASEB J 2004;18:816-27.

24. Burgess JK, Johnson PR, Ge Q, et al. Expression of connective tissue growth factor in asthmatic airway smooth muscle cells. Am J Respir Crit Care Med 2003;167:71-7.
25. Johnson PR, Burgess JK, Ge Q, et al. Connective tissue growth factor induces extracellular matrix in asthmatic airway smooth muscle. Am J Respir Crit Care Med 2006;173:32-41.

26. Been JV, Lugtenberg MJ, Smets E, et al. Preterm birth and childhood wheezing disorders: a systematic review and meta-analysis. PLoS Med 2014;11:e1001596.

27. Malmström K, Pelkonen AS, Mäkelä MJ. Remodeling, inflammation and airway responsiveness in early childhood asthma. Curr Opin Allergy Clin Immunol 2013;13:203-10.

28. Dekkers BG, Maarsingh H, Meurs H, Gosens R. Airway structural components drive airway smooth muscle remodeling in asthma. Proc Am Thorac Soc 2009;6:683-92.

29. Yee M, Chess PR, McGrath-Morrow SA, et al. Neonatal oxygen adversely affects lung function in adult mice without altering surfactant composition or activity. Am J Physiol Lung Cell Mol Physiol 2009;297:L641-9.

30. Salminen A, Paananen R, Vuolteenaho R, et al. Maternal endotoxininduced preterm birth in mice: fetal responses in toll-like receptors, collectins, and cytokines. Pediatr Res 2008;63:280-6.

31. Li MO, Wan YY, Sanjabi S, Robertson AK, Flavell RA. Transforming growth factor-beta regulation of immune responses. Annu Rev Immunol 2006;24:99-146.

32. Groneberg DA, Witt H, Adcock IM, Hansen G, Springer J. Smads as intracellular mediators of airway inflammation. Exp Lung Res 2004;30: 223-50.

33. Chen CM, Wang LF, Chou HC, Lang YD, Lai YP. Up-regulation of connective tissue growth factor in hyperoxia-induced lung fibrosis. Pediatr Res 2007;62:128-33.

34. Wu S, Capasso L, Lessa A, et al. High tidal volume ventilation activates Smad2 and upregulates expression of connective tissue growth factor in newborn rat lung. Pediatr Res 2008;63:245-50.

35. Britt RD Jr, Faksh A, Vogel ER, et al. Vitamin D attenuates cytokineinduced remodeling in human fetal airway smooth muscle cells. J Cell Physiol 2015;230:1189-98.

36. Vanoirbeek JA, Rinaldi M, De Vooght V, et al. Noninvasive and invasive pulmonary function in mouse models of obstructive and restrictive respiratory diseases. Am J Respir Cell Mol Biol 2010;42:96-104.

37. James AL, Hogg JC, Dunn LA, Paré PD. The use of the internal perimeter to compare airway size and to calculate smooth muscle shortening. Am Rev Respir Dis 1988;138:136-9.

38. Ochs M, Mühlfeld C. Quantitative microscopy of the lung: a problembased approach. Part 1: basic principles of lung stereology. Am J Physiol Lung Cell Mol Physiol 2013;305:L15-22.

39. Hsia CC, Hyde DM, Ochs M, Weibel ER; ATS/ERS Joint Task Force on Quantitative Assessment of Lung Structure. An official research policy statement of the American Thoracic Society/European Respiratory Society: standards for quantitative assessment of lung structure. Am J Respir Crit Care Med 2010;181:394-418.

40. Aravamudan B, VanOosten SK, Meuchel LW, et al. Caveolin-1 knockout mice exhibit airway hyperreactivity. Am J Physiol Lung Cell Mol Physiol 2012;303:L669-81. 